\title{
Acute macular neuroretinopathy: early receptor potential change suggests photoreceptor pathology
}

\author{
PAUL A. SIEVING, GERALD A. FISHMAN, THOMAS SALZANO, \\ AND MAURICE F. RABB
}

From the University of Illinois Eye and Ear Infirmary, Chicago, Illinois, USA

\begin{abstract}
SUMmary The early receptor potential (ERP) was recorded in a patient with typical fundus findings of unilateral acute macular neuroretinopathy. These amplitudes were compared with those of a normal control population. The ERP amplitude of the affected eye measured 13 months after onset of visual impairment was significantly reduced (4.8 SD) compared with the normal fellow eye. A subsequent ERP measurement 7 months later $(20$ months after onset $)$ showed the same amount of amplitude reduction, indicating that recovery had not occurred by this time. Since the ERP is generated during photolysis of visual pigment molecules and requires intact photoreceptor outer segment membranes to orient these molecules, our findings suggest that the outer segments are affected in at least some patients with this disorder. A retinal vascular aetiology for this disorder was rejected by studying a subject with a large retinal depression sign that occurred after a previous retinal vascular occlusion.
\end{abstract}

In 1975 Bos and Deutman' first described a retinal disorder that they called acute macular neuroretinopathy (AMNR). In their 4 patients this disease was characterised by mild visual impairment, paracentral scotomas, and ophthalmoscopically visible dark reddish wedge-shaped lesions in a parafoveal distribution. The lesions were located predominantly on the nasal side of the fovea. The optic disc, pigment epithelium, and retinal vessels did not show abnormal features by biomicroscopy or fluorescein angiography. An electroretinogram (ERG) performed on 2 patients was normal, as was an electro-oculogram (EOG) that was done on one patient. In 2 of their patients Bos and Deutman noted questionable dilatation of perifoveal capillaries without leakage. They believed that the disease process was localised superficially in the retinal layers.

Several similar cases, both unilateral and bilateral, were subsequently reported. ${ }^{2+}$ The dark reddish wedge-shaped lesions were mostly found on the nasal side of the fovea. ERG and EOG tests were normal. Priluck and colleagues ${ }^{3}$ noted a subtle macular hypofluorescence of the fluorescein angiogram, and they associated the fluorescein angiographic alterations

Correspondence to Dr P. A. Sieving. Department of Ophthalmology, U-490 University of California, San Francisco, San Francisco, California 94143, USA with that seen in retinal pigment epithelial or choriocapillaris disease. Whereas Bos and Deutman' believed that superficial retinal layers were involved, Priluck and coworkers suggested that the paracentral distribution of the lesions and the fact that the lesions were best seen with red-free light indicated deeper retinal involvement, specifically at the photoreceptor layer.

We measured the early receptor potential (ERP) from one patient who had monocular involvement with acute macular neuroretinopathy. ERP measurements provide a sensitive means for investigating pathology of the photoreceptors in vivo by electrophysiology. The ERP is generated at the level of the outer segments, as has been shown by microelectrode studies in animals. ${ }^{5}$ The human ERP action spectrum $^{\prime}$ and regeneration time ${ }^{7}$ have been correlated with visual pigment activity. The ERP response requires intact outer segment membranes to orient the visual photopigments." The ERP amplitude is linearly related to the number of photoisomerisations of visual pigment molecules within the photoreceptors. Since the photopigment molecules are an integral part of the photoreceptor outer segment membranes, these findings on the origin of the ERP provide the basis for correlating ERP amplitude reductions with changes at the level of the outer segments. 
Our patient had only one eye involved with acute macular neuroretinopathy; the fellow eye was normal by history and by examination. ERP amplitude differences between the patient's 2 eyes provided a sensitive indication of pathology in the involved eye. Since we record the ERP response from the posterior $53^{\circ}$ of the fundus, our ERP test is more sensitive to localised macular pathology than is a ganzfeld ERG, which records a mass response of the entire retina. In addition an ERG records complex neural events that approximate to a logarithmic response to visual pigment photoisomerisations. Since the ERP amplitude is linearly related to visual pigment density, the ERP is more sensitive to small changes in visual pigment density than is the ERG. Thus ERP measurements provide a means of investigating possible photoreceptor pathology in acute macular neuroretinopathy.

\section{Patient and methods}

A 20-year-old white woman was seen in February 1980 with a complaint of acutely decreased vision in the right eye for several days. Her past ocular history included an episode of 'iritis' of the right eye in October 1979, which resolved after 3 weeks of treatment with dilatation and topical steroids. No repeat episode had occurred, and the aetiology was not determined. Her systemic history and review of systems were noncontributory. She had no recent history of systemic illness or viral infection, and she had no known allergies. She was using an oral contraceptive (Orthonovum) for approximately one year. The family pedigree showed no relative with visual impairment.

Her best corrected acuity, in March 1980, was $6 / 12-2(20 / 40-2)$ OD with $-2 \cdot 50+0 \cdot 50 \times 90$ and $6 / 6$ $(20 / 20)$ OS with $-3 \cdot 00+0 \cdot 50 \times 90$. The external examination showed no abnormalities. Ocular motility was full. Lids, lashes, and lacrimal apparatus were normal. The sclera, conjunctiva, cornea, and anterior chamber were normal bilaterally by biomicroscopy. The pupils were equally round and reactive, with no afferent pupillary defect present. Both lenses showed a few pigment flecks on the anterior capsule and a few cortical flecks. Intraocular pressures were $12 \mathrm{mmHg}$ $\mathrm{OD}$ and $11 \mathrm{mmHg}$ OS.

Ophthalmoscopic examination of the right eye showed a normal-appearing disc and vessels. The macula contained 3 parafoveal wedge-shaped lesions on the nasal side of the fovea and a slight darkening of the foveola (Fig. 1). These lesions had a dark burntorange colour. The smooth internal limiting membrane reflex appeared disturbed over them. In continuity with the 3 parafoveal lesions was a further area superonasal to the fovea with a subtle, altered

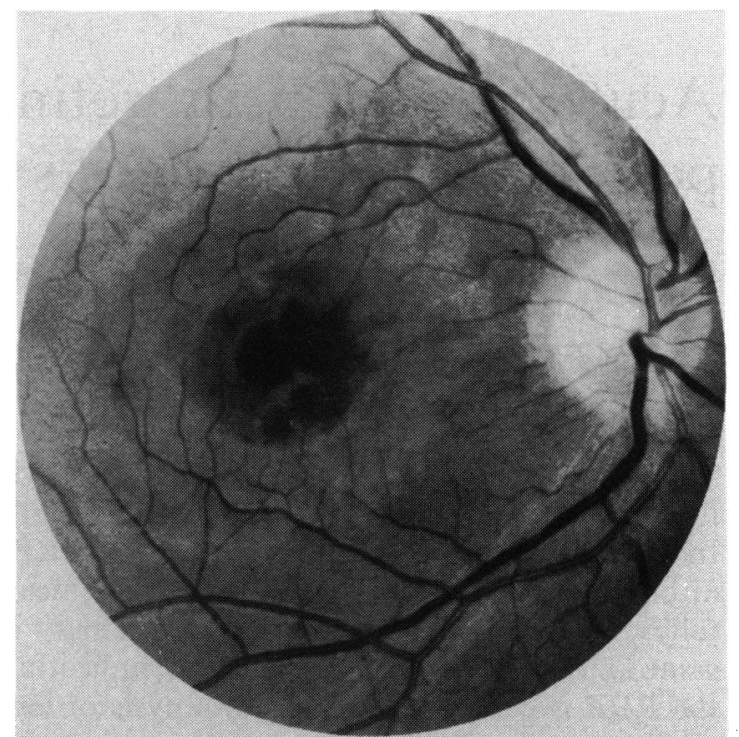

Fig. 1 Affected eye of patient with acute macular neuroretinopathy. Three burnt-orange lesions are noted in the nasal, parafoveal region.

reflex that gave the retina a slightly salmon coloured appearance. This larger lesion was about 3 disc diameters in extent. A fluorescein angiogram of the retina (Fig. 2) was normal, except for a subtle hypofluorescence in the region of the parafoveal lesions. No peripheral changes were seen either by oph-

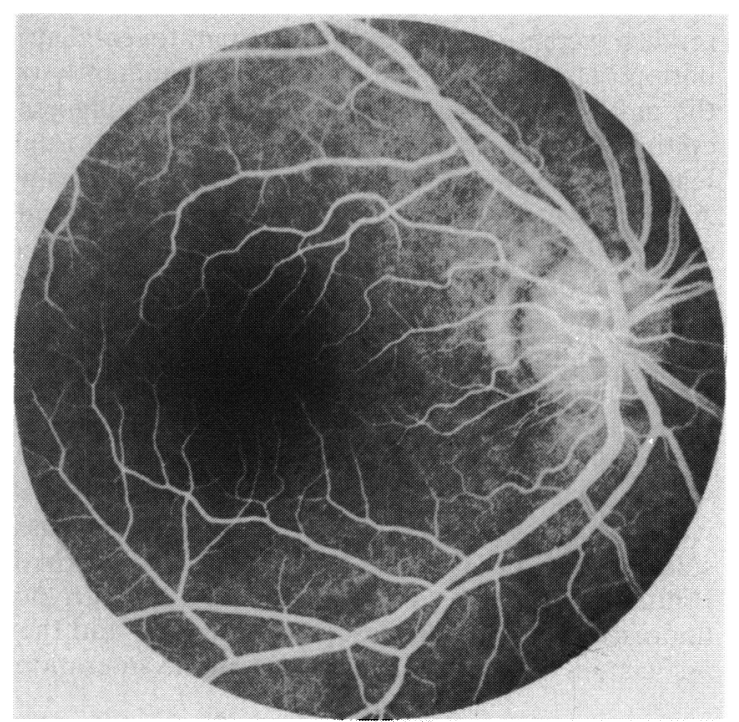

Fig. 2 Fluorescein angiogram of the affected eye in a patient with acute macular neuroretinopathy. A subtle hypofluorescence was seen in the affected region. 


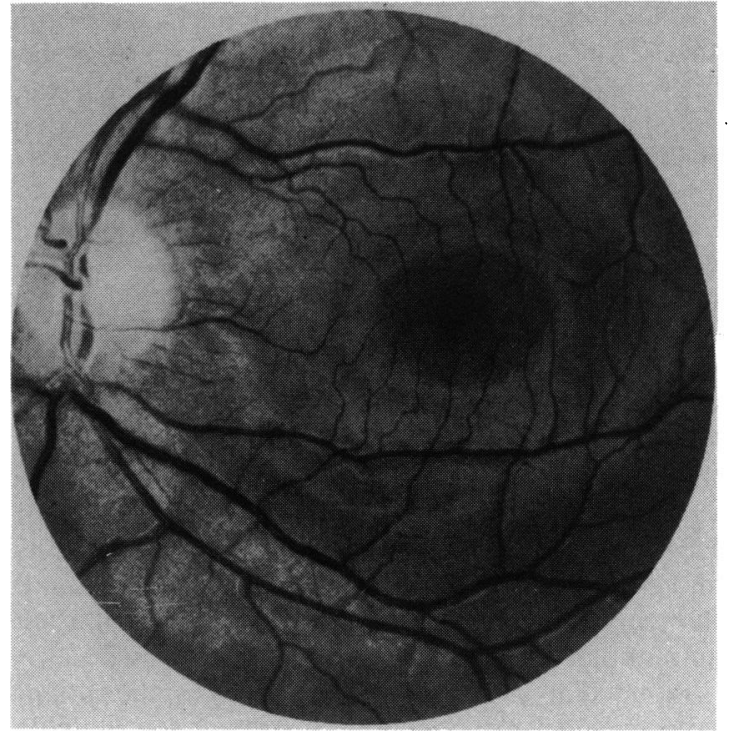

Fig. 3 Normal fellow eye of patient with unilateral acute macular neuroretinopathy.

thalmoscopy of fluorescein angiography. The left eye (Fig. 3) had a normal-appearing disc and vessels, and no macular or peripheral abnormalities were noted. A diagnosis of unilateral acute macular neuroretinopathy was made.

Visual field examination of the right eye with an Amsler grid (Fig. 4) showed 3 temporal paracentral scotomas, centred about $6^{\circ}$ from fixation, that cor-

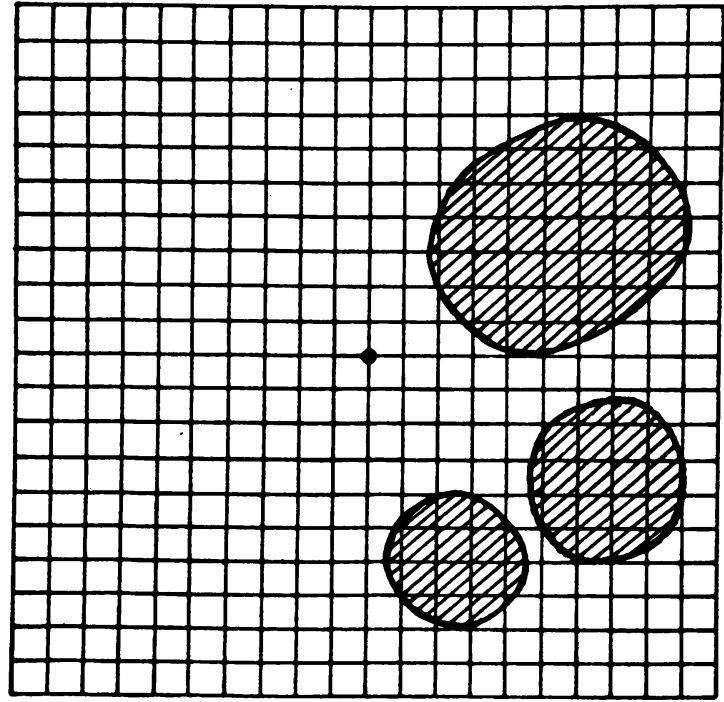

Fig. 4 Amsler grid central field of the affected eye shows 3 scotomata that correspond with the parafoveal lesions of acute macular neuroretinopathy.
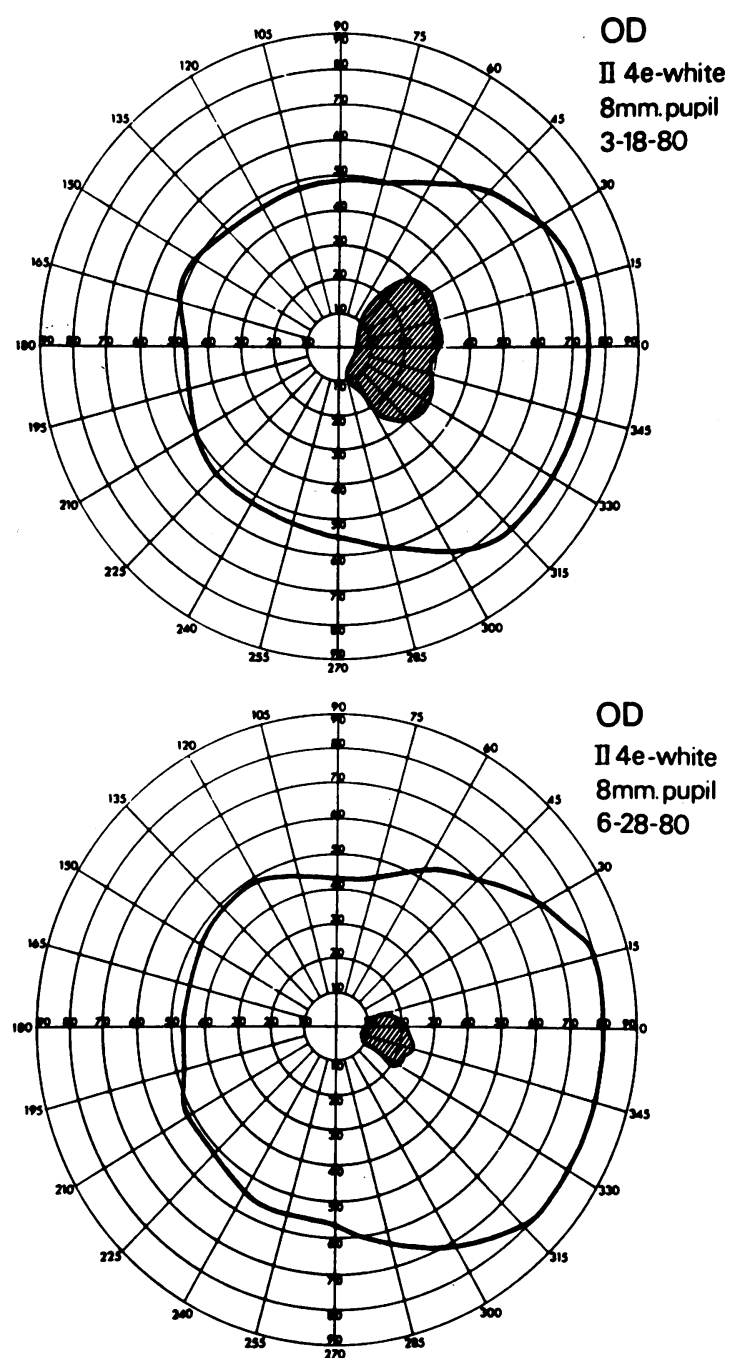

Fig. 5 Above: Goldmann visual field from patient's affected eye during the acute stage (18 March 1980). Below: On retesting 3 months later (28 June 1980) the defect was much smaller.

responded to the burnt-orange coloured parafoveal lesions. A Goldmann peripheral visual field of the right eye in March 1980 showed a wedge-shaped scotoma extending from $5^{\circ}$ to $30^{\circ}$ of visual angle with a II(4)e target (Fig. 5, upper). This defect corresponded to the macular area having the subtle, altered reflex. When peripheral field tests were repeated in June 1980, this larger macular field defect had begun to resolve (Fig. 5, lower), and only the 3 paracentral scotomata remained by 13 months after onset of symptoms. Her vision improved to $6 / 6-1$ (20/20-1) OD by June 1980 . The 3 parafoveal lesions remained visible by ophthalmoscopy, and 20 months 
after onset of symptoms they had not resolved. The left eye showed no visual field defects while the patient was followed up.

The ERP was measured with a Maxwellian view apparatus that subtended $53^{\circ}$ of visual angle and stimulated the posterior fundus region." A xenon flash stimulus had a 400$) \mu$ s duration at one-third peak height. Ultraviolet (WG-1) and infrared (KG-3) Schott glass filters were used, along with a Corning CS 3-71 filter, to limit the spectrum from 470 to 720 $\mathrm{nm}$. The intensity was $7 \cdot 7$ scotopic $\log \mathrm{td}-\mathrm{s}$ and $7 \cdot 4$ photopic log td-s (corrected for the Stiles-Crawford effect on cones from the $5.5 \mathrm{~mm}$ extended source image at the pupil). Pupils were maximally dilated with $10 \%$ phenylephrine hydrochloride, $1 \%$ tropicamide, and $1 \%$ cyclopentolate hydrochloride. The ERP signal was recorded at the cornea with a cotton wick electrode, and a differential signal input was supplied from the forehead and ear lobe. The preamplifier had a bandpass of $3 \mathrm{~Hz}$ to $30 \mathrm{kHz}(3 \mathrm{db}$ points). The signal was displayed on an oscilloscope and photographed. Subjects remained in room illumination for at least 30 minutes prior to the measurement without exposure to bright examining lights. Immediately prior to the test the subject was further dark-adapted for at least 15 minutes in ambient illumination of less than $\left(0 \cdot(2)\right.$ foot-lamberts $\left(0 \cdot() 7 \mathrm{~cd} / \mathrm{m}^{2}\right)$. This is sufficient to assure nearly complete regeneration of both rod and cone visual pigments. A further discussion of these ERP recording techniques is found elsewhere. "1"

The ERP amplitudes were measured from the isoelectric, prestimulus baseline to the trough of the R2 wave. This is the most stable portion of the recording, since the $\mathrm{R}$ I wave is a fast response of small amplitude. which is susceptible to noise. With the present apparatus we estimate that rod photoreceptors contribute between $23 \%$ and $49 \%$ of the R2 component of the ERP amplitude."

\section{Results}

The ERP was first measured in this patient in April 1981, 13 months after the onset of her visual symptoms. Three recordings were performed for each eye on this day. The amplitudes for the normal left eye were 173,173 , and $183 \mu \mathrm{v}$, with a mean of $176 \mu \mathrm{v}$. For the affected right eye, the amplitudes were 143, 143 , and $138 \mu \mathrm{v}$, with a mean of $141 \mu$. The mean reduction of amplitude of the affected right eye was $20 \%$ compared with the normal left eye.

The patient was re-examined 8 months later, in December 1981. The ERP amplitudes of the normal eye were 173 and $179 \mu \mathrm{v}$, with a mean of $176 \mu \mathrm{v}$. The affected eye had amplitudes of 126 and $144 \mu v$, with a mean of $135 \mu \mathrm{v}$. The $23 \%$ mean amplitude reduction in the affected eye was essentially unchanged from that recorded 7 months earlier. The $\mathrm{F}$ ratio (one-way analysis of variance using 5 data points for each eye, combined from both recording sessions) suggests that the amplitude difference between the eyes $(F(1,8)=$ $89 \cdot 8 ; \mathrm{p}<0 \cdot 001)$ is significant.

The ERP amplitudes of this patient were compared with those of a control population whose normal ERP data were obtained with the same apparatus during the interval that this patient was followed up. These normal data were also used in a separate study. ${ }^{12}$ The normal values reported below are of 2 kinds. First, ERP amplitudes were measured from the 2 eyes of a normal individual and the intraocular variation was noted. This intraocular variation of ERP amplitudes was determined for 9 normal subjects and the population mean is reported. We have found that this is a sensitive indicator of abnormal ERP amplitudes. However, it can be applied only in cases of monocular pathology, as was present for the patient reported on here. Secondly, a comparison is made with the mean ERP amplitude of a series of 12 normal subjects. These data are measurements either of a single eye of each subject or are an average of the 2 eyes (for 9 subjects). In either case only one value was attributed to each of the 12 normal persons.

The patient's eye affected with acute macular neuroretinopathy had a mean amplitude that was $21 \%$ less than her normal fellow eye $(n=5$ measurements). This $21 \%$ decrease is $4.8 \mathrm{SD}$ away from the mean difference between the 2 normal eyes of subjects in a control group (mean $=6 \cdot() \%, \mathrm{SD}=3 \cdot 1 \%, \mathrm{n}=9$ subjects; measured as the (high-low)/high value). The probability of finding a $21 \%$ variation between 2 normal eyes of the same individual would be quite small $(p<0 \cdot(0)(0) 1)$ in our control group. This strongly suggests that the ERP amplitude of this patient's affected eye is significantly reduced compared with her normal eye.

The patient's ERP amplitudes were also compared with the mean of a normal population. This provides a less sensitive criterion for abnormal ERP amplitudes than the first method because it is subject to increased variability of testing conditions and population heterogeneity. The affected eye had a mean amplitude of $139 \mu \mathrm{v}$ ( $\mathrm{n}=5$ measurements), which is $1.7 \mathrm{SD}$ below normal (normal mean $=180 \mu \mathrm{v}, \mathrm{SD}=23 \mu \mathrm{v}$, $\mathrm{n}=12$ subjects). The $176 \mu \mathrm{v}$ mean amplitude of the fellow eye was quite normal ( $n=5$ measurements).

The ERP waveform of the affected eye appeared nomal. The R1 amplitude was about $11 \%$ of the total $(\mathrm{R} 1+\mathrm{R} 2)$ ERP amplitude, which is within the usual range seen in our normal subjects. The $\mathrm{R} 1 \mathrm{com}$ ponent is generated primarily by cone photoreceptors and is absent in achromatic subjects. " Thus we cannot identify a selective dysfunction of cone photo- 
receptors for this patient on the basis of the ERP recordings.

\section{Discussion}

We measured the ERP of a subject with typical ophthalmoscopic findings of so-called acute macular neuroretinopathy. The ERP amplitude was significantly decreased in the affected eye compared with the normal eye. This suggests that the photoreceptor outer segments can be abnormal in this disease.

On our initial examination (March 1980) the visual field scotoma included about $30 \%$ of the posterior fundus area that we later tested with ERP measurements. By June 1980 this scotoma had decreased in size and included only about $5 \%$ of the region subtended by the ERP test flash. At the time of the first ERP measurements (April 1981) the scotoma was limited to the ophthalmoscopically visible parafoveal lesions. In this region of the fundus the area of involved retina has a nearly linear correspondence with an ERP test amplitude reduction. Thus if the photoreceptors in $5 \%$ of the test area were totally destroyed, one could expect approximately a 5\% reduction of the ERP amplitude.

The $21 \%$ mean ERP amplitude reduction that this patient showed suggests at least 2 models to understand the distribution of the pathology: (1) the photoreceptor outer segments might be absent in $21 \%$ of the region tested, or (2) the outer segment visual pigment density might be decreased by $21 \%$ throughout the entire test region (or a combination of both). If the photoreceptors were absent, an absolute scotoma would have been found for $21 \%$ of the ERP test area. The first ERP amplitude measurements were reduced by about $20 \%$, but only about $5 \%$ of the test region was scotomatous at that time. Thus the pathology was probably more diffuse than indicated either by the visual field scotoma or by the ophthalmoscopically visible lesions.

It is not surprising that visual pigment density might be decreased while the patient was aware of only minimal visual symptoms. Visual function, measured by most psychophysical tests such as dark adaptation, has an approximately logarithmic relationship with visual pigment density. Thus a $21 \%$ reduction of visual pigment density would raise the final dark adaptation threshold by only $0 \cdot 1 \log$ unit and would result in only minimally decreased visual function. Since the ERG amplitude is also a logarithmic function of pigment density, it might well be considered normal, as it was in the previously reported cases. ${ }^{1+4}$ However, the ERP amplitude, which is a linear function of visual pigment density, is more sensitive to a small decrease. In addition, our ERP stimulus subtends only the posterior portion of the fundus and therefore is more sensitive in identifying pathology limited to the macula than is a ganzfeld ERG stimulus that records activity throughout the entire retina.

Bos and Deutman' described an irregular reflex over the lesions in 3 of their 4 patients. Rush ${ }^{2}$ noted a 'gossamer-like quality' to the lesions. We noted an altered retinal reflex in our patient that appeared similar to the 'retinal depression sign' reported by Goldbaum. ${ }^{13}$ The depression sign is believed to be an alteration of the internal limiting membrane reflex due to focal tissue loss and retinal thinning. The internal limiting membrane dips down into the pit left by the thinned retina. The depression appears dark because light is reflected away from the observer. The original description of the retinal depression sign was associated with small retinal infarcts such as are found in sickle cell retinopathy and hypertensive retinopathy. ${ }^{13}$ Vascular occlusion and capillary nonperfusion may result in ischaemia with eventual nerve fibre layer necrosis and retinal thinning.

The similarity of AMNR lesions to small retinal depression sign alterations suggested a possible common aetiology. However, none of the reported AMNR cases was noted to have a fundus appearance classically found with an acute retinal ischaemic event, such as tissue oedema or exudates. In addition pathology limited to a retinal vascular aetiology is probably not consistent with a decreased ERP amplitude; Yonemura and Kawasaki ${ }^{14}$ found that the ERP amplitude is not reduced after a central retinal artery occlusion.

To assess further a possible common retinal vascular aetiology of AMNR and retinal depression signs we measured the ERP from one patient with a monocular retinal depression sign. This test subject had haemoglobin sickle cell disease and presented with parafoveal retinal ischaemia and exudation in one eye. Follow-up examinations showed a large macular retinal depression sign in the area of the previous vascular occlusion. Nerve fibre atrophy was seen at the optic disc. This patient had a visual field defect that affected about $30 \%$ of the area of the ERP test region and in this regard was similar to that of our AMNR patient's initial presentation. The fellow eye was ophthalmoscopically normal and had no visual field defect in the ERP test region. We recorded an ERP from both eyes about a year after the occlusion has resolved and the retina was reperfused. If the photoreceptors had been destroyed in the region of the retinal ischaemia, a 30\% ERP amplitude difference should have been found between the eyes, on the basis of the visual field defect. However, only a $1 \%$ difference of ERP amplitude was recorded between the 2 eyes (measured approximately one year after the acute event). This is not unexpected, 
since the retinal vessels are not thought to provide a significant vascular supply for the photoreceptors. The suggestion that an acute lesion at one retinal level may later affect other retinal layers ${ }^{4}$ does not appear to be valid for retinal vascular disease in this sickle cell patient or, by extension, for our AMNR patient. Even though the parafoveal lesions of AMNR may appear similar to lesions of retinal depression signs, a retinal vascular aetiology does not explain the ERP findings in our patient with acute macular neuroretinopathy.

This work was supported in part by Core Grant EY 1792 and Training Grant EY7038 from the National Eyc Institute, to the Department of Ophthalmology, University of Illinois Eyc and Ear Infirmary; by a grant from the National Retinitis Pigmentosa Foundation, Baltimore, Maryland (Dr Fishman); and by a grant from Fight For Sight, New York (Dr Sicving). The authors thank Dr Morton $F$ Goldberg and Maxine Gere, who read the manuscript.

\section{References}

I Bos PJ, Deutman AF. Acutc macular ncurorctinopathy. A m J Ophthalmol 1975; 80: 573-84.

2 Rush JA. Acute macular neuroretinopathy. Am J Ophthalmol 1977: 83: 49()-3.
3 Priluck IA, Bucttner H, Roberton, D. Acutc macular neuroretinopathy. Am J Ophthalmol 1978; 86: 775-8.

4 Neetens A. Burvenich H. Presumed inflammatory maculopathics. Trans Ophthalmol Soc UK 1978; 98: 160)-6.

5 Brown KT, Watanabe K. Murakami M. The carly and late receptor potentials of monkey concs and rods. Cold Spring Harbor Symp Quant Biol 1965; 30: 457-82.

6 Carr RE, Sicgel IM. Action spectrum of the human carly receptor potential. Nature 1970); 225: 88-9.

7 Sieving PA, Fishman GA. Regeneration of the human early receptor potential (ERP) following prolonged and flash bleaches. Invest Ophthalmol Visual Sci 1980); 20 (suppl): 260).

8 Cone RA, Brown PK. Dependence of the early receptor potential on the orientation of rhodopsin. Science 1967; 156: 536 .

9 Hodgkin AL, O'Bryan PM. Internal recording of the carly receptor potential in turtle cones. J Physiol 1977; 267: 737-66.

10) Sicving PA. In vivo determination of visual pigment regeneration in humans by measurements of the early receptor potential (ERP). PhD thesis. Chicago: University of Illinois at the Medical Center. 1981.

II Sicving PA, Fishman GA. Rod contribution to the human carly receptor potential (ERP) estimated from monochromats' data. Doc Ophthalmol Proc Ser 1982; 31: 95-102.

12 Sieving PA, Fishman GA, Alexander KR, Goldberg MF. Early receptor potential (ERP) measurements in human ocular sidcrosis. Arch Ophthalmol 1983; 101: 1716-20.

13 Goldbaum MH. Retinal depression sign indicating a small infarct. Ain J Ophthalmol 1978; 86: 45-5.

14 Yonemura D, Kawasaki K. The carly receptor potential in the human clectroretinogram. Jpn J Physiol 1967; 17: 235-44. 